\title{
Research Article \\ Vartiational Optimal-Control Problems with Delayed Arguments on Time Scales
}

\author{
Thabet Abdeljawad (Maraaba), ${ }^{1}$ Fahd Jarad, ${ }^{1}$ \\ and Dumitru Baleanu, ${ }^{1,2}$ \\ ${ }^{1}$ Department of Mathematics and Computer Science, Çankaya University, 06530 Ankara, Turkey \\ ${ }^{2}$ Institute of Space Sciences, P.O. BOX MG-23, 76900 Magurele-Bucharest, Romania \\ Correspondence should be addressed to Thabet Abdeljawad (Maraaba), thabet@cankaya.edu.tr
}

Received 11 August 2009; Revised 3 November 2009; Accepted 16 November 2009

Recommended by Paul Eloe

This paper deals with variational optimal-control problems on time scales in the presence of delay in the state variables. The problem is considered on a time scale unifying the discrete, the continuous, and the quantum cases. Two examples in the discrete and quantum cases are analyzed to illustrate our results.

Copyright (C) 2009 Thabet Abdeljawad (Maraaba) et al. This is an open access article distributed under the Creative Commons Attribution License, which permits unrestricted use, distribution, and reproduction in any medium, provided the original work is properly cited.

\section{Introduction}

The calculus of variations interacts deeply with some branches of sciences and engineering, for example, geometry, economics, electrical engineering, and so on [1]. Optimal control problems appear in various disciplines of sciences and engineering as well [2].

Time-scale calculus was initiated by Hilger (see [3] and the references therein) being in mind to unify two existing approaches of dynamic models difference and differential equations into a general framework. This kind of calculus can be used to model dynamic processes whose time domains are more complex than the set of integers or real numbers [4]. Several potential applications for this new theory were reported (see, e.g., [4-6] and the references therein). Many researchers studied calculus of variations on time scales. Some of them followed the delta approach and some others followed the nabla approach (see, e.g., [7-12]).

It is well known that the presence of delay is of great importance in applications. For example, its appearance in dynamic equations, variational problems, and optimal control problems may affect the stability of solutions. Very recently, some authors payed the attention to the importance of imposing the delay in fractional variational problems [13]. The nonlocality of the fractional operators and the presence of delay as well may give better results for problems involving the dynamics of complex systems. To the best of our 
knowledge, there is no work in the direction of variational optimal-control problems with delayed arguments on time scales.

Our aim in this paper is to obtain the Euler-Lagrange equations for a functional, where the state variables of its Lagrangian are defined on a time scale whose backward jumping operator is $\rho(t)=q t-h, q>0, h \geq 0$. This time scale, of course, absorbs the discrete, the continuous and the quantum cases. The state variables of this Lagrangian allow the presence of delay as well. Then, we generalize the results to the $n$-dimensional case. Dealing with such a very general problem enables us to recover many previously obtained results [14-17].

The structure of the paper is as follows. In Section 2 basic definitions and preliminary concepts about time scale are presented. The nabla time-scale derivative analysis is followed there. In Section 3 the Euler-Lagrange equations into one unknown function and then in the $n$-dimensional case are obtained. In Section 4 the variational optimal control problem is proposed and solved. In Section 5 the results obtained in the previous sections are particulary studied in the discrete and quantum cases, where two examples are analyzed in details. Finally, Section 6 contains our conclusions.

\section{Preliminaries}

A time scale is an arbitrary closed subset of the real line $\mathbb{R}$. Thus the real numbers and the natural numbers, $\mathbb{N}$, are examples of a time scale. Throughout this paper, and following [4], the time scale will be denoted by $\mathbb{T}$. The forward jump operator $\sigma: \mathbb{T} \rightarrow \mathbb{T}$ is defined by

$$
\sigma(t):=\inf \{s \in \mathbb{T}: s>t\}
$$

while the backward jump operator $\rho: \mathbb{T} \rightarrow \mathbb{T}$ is defined by

$$
\rho(t):=\sup \{s \in \mathbb{T}: s<t\}
$$

where, $\inf \emptyset=\sup \mathbb{T}$ (i.e., $\sigma(t)=t$ if $\mathbb{T}$ has a maximum $t$ ) and $\sup \emptyset=\inf \mathbb{T}$ (i.e., $\rho(t)=t$ if $\mathbb{T}$ has a minimum $t$ ). A point $t \in \mathbb{T}$ is called right-scattered if $t<\sigma(t)$, left-scattered if $\rho(t)<t$, and isolated if $\rho(t)<t<\sigma(t)$. In connection we define the backward graininess function $v: \mathbb{T} \rightarrow[0, \infty)$ by

$$
v(t)=t-\rho(t)
$$

In order to define the backward time-scale derivative down, we need the set $\mathbb{T}_{\kappa}$ which is derived from the time scale $\mathbb{T}$ as follows. If $\mathbb{T}$ has a right-scattered minimum $m$, then $\mathbb{T}_{\kappa}=$ $\mathbb{T}-\{m\}$. Otherwise, $\mathbb{T}_{\kappa}=\mathbb{T}$.

Definition 2.1 (see [18]). Assume that $f: \mathbb{T} \rightarrow \mathbb{R}$ is a function and $t \in \mathbb{T}_{\kappa}$. Then the backward time-scale derivative $f^{\nabla}(t)$ is the number (provided that it exists) with the property that given any $\epsilon>0$, there exists a neighborhood $U$ of $t$ (i.e., $U=(t-\delta, t+\delta)$ for some $\delta>0)$ such that

$$
|[f(s)-f(\rho(t))]-[s-\rho(t)]| \leq \epsilon|s-\rho(t)| \quad \forall s \in U .
$$

Moreover, we say that $f$ is (nabla) differentiable on $\mathbb{T}_{\kappa}$ provided that $f^{\nabla}(t)$ exists for all $t \in \mathbb{T}_{\kappa}$. 
The following theorem is Theorem 3.2 in [19] and an analogue to Theorem 1.16 in [4].

Theorem 2.2 (see [18]). Assume that $f: \mathbb{T} \rightarrow \mathbb{R}$ is a function and $t \in \mathbb{T}_{\kappa}$, then one has the following.

(i) If $f$ is differentiable at then $f$ is continuous at $t$.

(ii) If $f$ is continuous at $t$ and $t$ is left-scattered, then $f$ is differentiable at $t$ with

$$
f^{\nabla}(t)=\frac{f(t)-f(\rho(t))}{\mathcal{v}(t)}
$$

(iii) If $t$ is left-dense, then $f$ is differentiable at $t$ if and only if the limit

$$
\lim _{s \rightarrow t} \frac{f(t)-f(s)}{t-s}
$$

exists as a finite number. In this case

$$
f^{\nabla}(t)=\lim _{s \rightarrow t} \frac{f(t)-f(s)}{t-s}
$$

(iv) If $f$ is $\nabla$-differentiable at $t$, then

$$
f(t)=f(\rho(t))+v(t) f^{\nabla}(t)
$$

Example 2.3. (i) $\mathbb{T}=\mathbb{R}$ or any any closed interval (the continuous case) $\sigma(t)=\rho(t)=t$, $v(t)=0$, and $f^{\nabla}(t)=f^{\prime}(t)$.

(ii) $\mathbb{T}=h \mathbb{Z}, h>0$ or any subset of it (the difference calculus, a discrete case) $\sigma(t)=t+h$, $\rho(t)=t-h, v(t)=h$, and $f^{\nabla}(t)=\nabla_{h} f(t)=f(t)-f(t-h)$.

(iii) $\mathbb{T}=\mathbb{T}_{q}=\left\{q^{n}: n \in \mathbb{Z}\right\} \cup\{0\}, 0<q<1$, (quantum calculus) $\sigma(t)=q^{-1} t, \rho(t)=q t$, $v(t)=(1-q) t$, and $f^{\nabla}(t)=\nabla_{q} f(t)=(f(t)-f(q t)) /(1-q) t$.

(iv) $\mathbb{T}=\mathbb{T}_{q}^{h}=\left\{q^{k}-\sum_{i=0}^{k-2} q^{i} h: k \geq 2, k \in \mathbb{N}\right\} \cup\{-h /(1-q)\}, 0<q<1, h>0$ (unifying the difference calculus and quantum calculus). There are $\sigma(t)=q^{-1}(t+h), \rho(t)=q t-h$, $v(t)=(1-q) t+h$, and $f^{\nabla}(t)=\nabla_{q}^{h} f(t)=(f(t)-f(q t-h)) /((1-q) t+h)$. If $\alpha_{0} \in \mathbb{N}$ then $\rho^{\alpha_{0}}(t)=$ $q^{\alpha_{0}} t-\sum_{k=0}^{\alpha_{0}-1} q^{k} h$ and so $\nabla_{q}^{h} \rho^{\alpha_{0}}(t)=q^{\alpha_{0}}$. Note that in this example the backward operator is of the form $\rho(t)=c t+d$ and hence $\mathbb{T}_{q}^{h}$ is an element of the class $H$ of time scales that contains the discrete, the usual, and the quantum calculus (see [17]).

Theorem 2.4. Suppose that $f, g: \mathbb{T} \rightarrow \mathbb{R}$ are nabla differentiable at $t \in \mathbb{T}_{\kappa}$, then,

(1) the sum $f+g: \mathbb{T} \rightarrow \mathbb{R}$ is nabla differentiable at $t$ and $(f+g)^{\nabla}(t)=f^{\nabla}(t)+g^{\nabla}(t)$;

(2) for any $\lambda \in \mathbb{R}$, the function $\lambda f: \mathbb{T} \rightarrow \mathbb{R}$ is nabla differentiable at $t$ and $(\lambda f)^{\nabla}(t)=\lambda f^{\nabla}(t)$;

(3) the product $f g: \mathbb{T} \rightarrow \mathbb{R}$ is nabla differentiable at $t$ and

$$
(f g)^{\nabla}=f^{\nabla}(t) g(t)+f^{\rho}(t) g^{\nabla}(t)=f^{\nabla}(t) g^{\rho}(t)+f(t) g^{\nabla}(t) .
$$


For the proof of the following lemma we refer to [20].

Lemma 2.5. Let $\mathbb{T}$ be an $H$-time scale (in particular $\mathbb{T}=\mathbb{T}_{q}^{h}$ ), $f: \mathbb{T} \rightarrow \mathbb{R}$ two times nabla differentiable function, and $g(t)=\rho^{\alpha_{0}}(t)$, for $\alpha_{0} \in \mathbb{N}$. Then

$$
(f \circ g)^{\nabla}(t)=f^{\nabla}(g(t)) \cdot g^{\nabla}(t), \quad t \in \mathbb{T}_{\mathcal{K}}
$$

Throughout this paper we use for the time-scale derivatives and integrals the symbol $\nabla_{q}^{h}$ which is inherited from the time scale $\mathbb{T}_{q}^{h}$. However, our results are true also for the $H$ time scales (those time scales whose jumping operators have the form $a t+b$ ). The time scale $\mathbb{T}_{q}^{h}$ is a natural example of an $H$-time scale.

Definition 2.6. A function $F: \mathbb{T} \rightarrow \mathbb{R}$ is called a nabla antiderivative of $f: T \rightarrow \mathbb{R}$ provided $F(t)=f(t)$, for all $t \in \mathbb{T}_{\kappa}$. In this case, for $a, b \in \mathbb{T}$, we write

$$
\int_{a}^{b} f(t) \nabla t:=F(b)-F(a)
$$

The following lemma which extends the fundamental lemma of variational analysis on time scales with nabla derivative is crucial in proving the main results.

Lemma 2.7. Let $g \in C_{\mathrm{ld}}, g:[a, b] \rightarrow \mathbb{R}^{n}$. Then

$$
\int_{a}^{b} g^{T}(t) \eta^{\nabla}(t) \nabla t \quad \forall \eta \in C_{\mathrm{ld}}^{1} \text { with } \eta(a)=\eta(b)=0
$$

holds if and only if

$$
g(t) \equiv c \quad \text { on }[a, b]_{\kappa} \text { for some } c \in \mathbb{R}^{n} .
$$

The proof can be achieved by following as in the proof of Lemma 4.1 in [9] (see also [17]).

\section{First-Order Euler-Lagrange Equation with Delay}

We consider the $\mathbb{T}_{q}^{h}$-integral functional $J: S \rightarrow \mathbb{R}$,

$$
J(y)=\int_{a}^{b} L\left(x, y^{\rho}(x), \nabla_{q}^{h} y(x), y^{\rho}\left(\rho^{\alpha_{0}}(x)\right), \nabla_{q}^{h} y\left(\rho^{\alpha_{0}}(x)\right)\right) \nabla_{q}^{h} x,
$$


where

$$
\begin{gathered}
a, b \in \mathbb{T}_{q^{\prime}}^{h} \quad a<\rho^{\alpha_{0}}(b)<b, \\
L:[a, b] \times\left(\mathbb{R}^{n}\right)^{4} \longrightarrow \mathbb{R}, \quad y^{\rho}(x)=y(\rho(x)), \\
S=\left\{y:\left[\rho^{\alpha_{0}}(a), b\right] \longrightarrow \mathbb{R}^{n}: y(x)=\varphi(x)\left(\forall x \in\left[\rho^{\alpha_{0}}(a), a\right]\right), y(b)=c_{0}\right\} .
\end{gathered}
$$

We will shortly write

$$
L(x) \equiv L\left(x, y^{\rho}(x), \nabla_{q}^{h} y(x), y^{\rho}\left(\rho^{\alpha_{0}}(x)\right), \nabla_{q}^{h} y\left(\rho^{\alpha_{0}}(x)\right)\right)
$$

We calculate the first variation of the functional $J$ on the linear manifold $S$. Let $\eta \in$ $H=\left\{h:\left[\rho^{\alpha_{0}}(a), b\right] \rightarrow \mathbb{R}^{n}: h(x)=0\left(\forall x \in\left[\rho^{\alpha_{0}}(a), a\right] \cup\{b\}\right)\right\}$, then

$$
\begin{gathered}
\delta J(y(x), \eta(x))=\left.\frac{d}{d \epsilon} J(y(x)+\epsilon \eta(x))\right|_{\epsilon=0} \\
\int_{a}^{b}\left[\partial_{1} L(x) \eta^{\rho}(x)+\partial_{2} L(x) \nabla_{q}^{h} \eta(x)+\partial_{3} L(x) \eta^{\rho}\left(\rho^{\alpha_{0}}(x)\right)+q^{\alpha_{0}} \partial_{4} L(x) \nabla_{q}^{h} \eta\left(\rho^{\alpha_{0}}(x)\right)\right] \nabla_{q}^{h} x,
\end{gathered}
$$

where

$$
\partial_{1} L=\frac{\partial L}{\partial\left(y^{\rho}(x)\right)}, \quad \partial_{2} L=\frac{\partial L}{\partial\left(\nabla_{q}^{h} y(x)\right)}, \quad \partial_{3} L=\frac{\partial L}{\partial\left(y^{\rho}\left(\rho^{\alpha_{0}}(x)\right)\right)}, \quad \partial_{4} L=\frac{\partial L}{\partial\left(\nabla_{q}^{h} y\left(\rho^{\alpha_{0}}(x)\right)\right)},
$$

and where Lemma 2.5 and that $\nabla_{q}^{h} \rho^{\alpha_{0}}(t)=q^{\alpha_{0}}$ are used. If we use the change of variable $u=\rho^{\alpha_{0}}(x)$, which is a linear function, and make use of Theorem 1.98 in [4] and Lemma 2.5 we then obtain

$$
\begin{aligned}
\delta J(y(x), \eta(x))= & \int_{a}^{b}\left[\partial_{1} L(x) \eta^{\rho}(x)+\partial_{2} L(x) \nabla_{q}^{h} \eta(x)\right] \nabla_{q}^{h} x \\
& +\int_{a}^{\rho^{\alpha_{0}}(b)}\left[q^{-\alpha_{0}} \partial_{3} L\left(\left(\rho^{\alpha_{0}}\right)^{-1}(x)\right) \eta^{\rho}(x)+q^{-\alpha_{0}} \partial_{4} L\left(\left(\rho^{\alpha_{0}}\right)^{-1}(x)\right) \nabla_{q}^{h} \eta(x)\right] \nabla_{q}^{h} x,
\end{aligned}
$$

where we have used the fact that $\eta \equiv 0$ on $\left[\rho^{\alpha_{0}}(a), a\right]$. 
Splitting the first integral in (3.6) and rearranging will lead to

$$
\begin{gathered}
\delta J(y(x), \eta(x))=\int_{a}^{\rho^{\alpha_{0}}(b)}\left[\partial_{1} L(x) \eta^{\rho}(x)+\partial_{2} L(x) \nabla_{q}^{h} \eta(x)+q^{-\alpha_{0}} \partial_{3} L\left(\left(\rho^{\alpha_{0}}\right)^{-1}(x)\right) \eta^{\rho}(x)\right. \\
\left.\quad+q^{-\alpha_{0}} \partial_{4} L\left(\left(\rho^{\alpha_{0}}\right)^{-1}(x)\right) \nabla_{q}^{h} \eta(x)\right] \nabla_{q}^{h} x \\
+\int_{\rho^{\alpha_{0}(b)}}^{b}\left[\partial_{1} L(x) \eta^{\rho}(x)+\partial_{2} L(x) \nabla_{q}^{h} \eta(x)\right] \nabla_{q}^{h} x .
\end{gathered}
$$

If we make use of part (3) of Theorem 2.4 then we reach

$$
\begin{aligned}
& \delta J(y(x), \eta(x)) \\
& \begin{aligned}
=\int_{a}^{\rho^{\alpha_{0}}(b)}\left\{\partial_{2} L(x) \nabla_{q}^{h} \eta(x)+q^{-\alpha_{0}} \partial_{4} L\left(\left(\rho^{\alpha_{0}}\right)^{-1}(x)\right) \nabla_{q}^{h} \eta(x)+\nabla_{q}^{h}\left[\int_{a}^{x} \partial_{1} L(z) \nabla_{q}^{h} z \cdot \eta(x)\right]\right. \\
\quad-\int_{a}^{x} \partial_{1} L(z) \nabla_{q}^{h} z \cdot \nabla_{q}^{h} \eta(x)+q^{-\alpha_{0}} \nabla_{q}^{h}\left[\int_{a}^{x} \partial_{3} L\left(\left(\rho^{\alpha_{0}}\right)^{-1}(z)\right) \nabla_{q}^{h} z \cdot \eta(x)\right] \\
\left.\quad-q^{-\alpha_{0}} \int_{a}^{x} \partial_{3} L\left(\left(\rho^{\alpha_{0}}\right)^{-1}(z)\right) \nabla_{q}^{h} z \cdot \nabla_{q}^{h} \eta(x)\right\} \nabla_{q}^{h} x \\
+\int_{\rho^{\alpha_{0}(b)}}^{b}\left\{\partial_{2} L(x) \nabla_{q}^{h} \eta(x)+\nabla_{q}^{h}\left[\int_{\rho^{\alpha_{0}}(b)}^{x} \partial_{1} L(z) \nabla_{q}^{h} z \cdot \eta(x)\right]-\int_{\rho^{\alpha_{0}(b)}}^{x} \partial_{1} L(z) \nabla_{q}^{h} z \cdot \nabla_{q}^{h} \eta(x)\right\} \nabla_{q}^{h} x .
\end{aligned}
\end{aligned}
$$

In (3.8), once choose $\eta$ such that $\eta(a)=0$ and $\eta \equiv 0$ on $\left[q^{\alpha_{0}} b, b\right]$ and in another case choose $\eta$ such that $\eta(b)=0$ and $\eta \equiv 0$ on $\left[a, q^{\alpha_{0}} b\right]$, and then make use of Lemma 2.7 to arrive at the following theorem.

Theorem 3.1. Let $J: S \rightarrow \mathbb{R}$ be the $\mathbb{T}_{q}^{h}$-integral functional

$$
J(y)=\int_{a}^{b} L\left(x, y^{\rho}(x), \nabla_{q}^{h} y(x), y^{\rho}\left(\rho^{\alpha_{0}}(x)\right), \nabla_{q}^{h} y\left(\rho^{\alpha_{0}}(x)\right)\right) \nabla_{q}^{h} x
$$

where

$$
\begin{gathered}
a, b \in \mathbb{T}_{q^{\prime}}^{h} \quad a<\rho^{\alpha_{0}}(b)<b, \\
L:[a, b] \times\left(\mathbb{R}^{n}\right)^{4} \longrightarrow \mathbb{R}, \quad y^{\rho}(x)=y(\rho(x)), \\
S=\left\{y:\left[\rho^{\alpha_{0}}(a), b\right] \longrightarrow \mathbb{R}^{n}: y(x)=\varphi(x)\left(\forall x \in\left[\rho^{\alpha_{0}}(a), a\right]\right), y(b)=c_{0}\right\} .
\end{gathered}
$$


Then the necessary condition for $J(y)$ to possess an extremum for a given function $y(x)$ is that $y(x)$ satisfies the following Euler-Lagrange equations

$$
\begin{gathered}
\nabla_{q}^{h} \partial_{2} L(x)+q^{-\alpha_{0}} \nabla_{q}^{h} \partial_{4} L\left(\left(\rho^{\alpha_{0}}\right)^{-1}(x)\right)=\partial_{1} L(x)+q^{-\alpha_{0}} \partial_{3} L\left(\left(\rho^{\alpha_{0}}\right)^{-1}(x)\right) \quad\left(x \in\left[a, \rho^{\alpha_{0}}(b)\right]_{\kappa}\right), \\
\nabla_{q}^{h} \partial_{2} L(x)=\partial_{1} L(x) \quad\left(x \in\left[\rho^{\alpha_{0}}(b), b\right]_{\kappa}\right) .
\end{gathered}
$$

Furthermore, the equation:

$$
\left.q^{-\alpha_{0}} \partial_{4} L\left(\left(\rho^{\alpha_{0}}\right)^{-1}(x)\right) \eta(x)\right|_{a} ^{\rho^{\alpha_{0}}(b)}=0
$$

holds along $y(x)$ for all admissible variations $\eta(x)$ satisfying $\eta(x)=0, x \in\left[\rho^{\alpha_{0}}(a), a\right] \cup\{b\}$.

The necessary condition represented by (3.12) is obtained by applying integration by parts in (3.7) and then substituting (3.11) in the resulting integrals. The above theorem can be generalized as follows.

Theorem 3.2. Let $J: S^{m} \rightarrow \mathbb{R}$ be the $\mathbb{T}_{q}^{h}$-integral functional

$$
\begin{aligned}
J\left(y_{1}, y_{2}, \ldots, y_{m}\right)=\int_{a}^{b} L( & \left(x, y_{1}^{\rho}(x), y_{2}^{\rho}(x), \ldots, y_{m}^{\rho}(x)\right),\left(\nabla_{q}^{h} y_{1}(x), \nabla_{q}^{h} y_{2}(x), \ldots, \nabla_{q}^{h} y_{m}(x)\right), \\
& \left(y_{1}^{\rho}\left(\rho^{\alpha_{0}}(x)\right), y_{2}^{\rho}\left(\rho^{\alpha_{0}}(x)\right), \ldots, y_{m}^{\rho}\left(\rho^{\alpha_{0}}(x)\right)\right), \\
& \left.\left(\nabla_{q}^{h} y_{1}\left(\rho^{\alpha_{0}}(x)\right), \nabla_{q}^{h} y_{1}\left(\rho^{\alpha_{0}}(x)\right), \ldots, \nabla_{q}^{h} y_{m}\left(\rho^{\alpha_{0}}(x)\right) \nabla_{q}^{h} x\right)\right),
\end{aligned}
$$

where

$$
\begin{gathered}
a, b \in \mathbb{T}_{q}^{h} \quad a<\rho^{\alpha_{0}}(b)<b, \\
L:[a, b] \times\left(\mathbb{R}^{n}\right)^{4 m} \longrightarrow \mathbb{R}, \quad y^{\rho}(x)=y(\rho(x)), \\
S^{m}=\left\{y=\left(y_{1}, y_{2}, \ldots, y_{m}\right): y_{i}:\left[\rho^{\alpha_{0}}(a), b\right] \longrightarrow \mathbb{R}^{n}, y_{i}(x)=\varphi_{i}(x)\right. \\
\left.\left(\forall x \in\left[\rho^{\alpha_{0}}(a), a\right]\right), y_{i}(b)=c_{i}, i=1,2, \ldots, m\right\} .
\end{gathered}
$$

Then a necessary condition for $J(y)$ to possess an extremum for a given function $y(x)=$ $\left(y_{1}(x), y_{2}(x), \ldots, y_{m}(x)\right)$ is that $y(x)$ satisfies the following Euler-Lagrange equations:

$$
\begin{gathered}
\nabla_{q}^{h} \partial_{2} L^{i}(x)+q^{-\alpha_{0}} \nabla_{q}^{h} \partial_{4} L^{i}\left(\left(\rho^{\alpha_{0}}\right)^{-1}(x)\right) \\
=\partial_{1} L^{i}(x)+q^{-\alpha_{0}} \partial_{3} L^{i}\left(\left(\rho^{\alpha_{0}}\right)^{-1}(x)\right) \quad\left(x \in\left[a, \rho^{\alpha_{0}}(b)\right]_{\kappa}\right), \\
\nabla_{q}^{h} \partial_{2} L^{i}(x)=\partial_{1} L^{i}(x) \quad\left(x \in\left[\rho^{\alpha_{0}}(b), b\right]_{\kappa}\right) .
\end{gathered}
$$


Furthermore, the equations

$$
\left.q^{-\alpha_{0}} \partial_{4} L^{i}\left(\left(\rho^{\alpha_{0}}\right)^{-1}(x)\right) \eta_{i}(x)\right|_{a} ^{\rho^{\alpha_{0}}(b)}=0
$$

hold along $y(x)$ for all admissible variations $\eta_{i}(x)$ satisfying

$$
\eta_{i}(x)=0, \quad x \in\left[\rho^{\alpha_{0}}(a), a\right] \cup\{b\}, i=1,2, \ldots, m,
$$

where

$\partial_{1} L^{i}=\frac{\partial L}{\partial\left(y_{i}^{\rho}(x)\right)}, \quad \partial_{2} L^{i}=\frac{\partial L}{\partial\left(\nabla_{q}^{h} y_{i}(x)\right)}, \quad \partial_{3} L^{i}=\frac{\partial L}{\partial\left(y_{i}^{\rho}\left(\rho^{\alpha_{0}}(x)\right)\right)}, \quad \partial_{4} L^{i}=\frac{\partial L}{\partial\left(\nabla_{q}^{h} y_{i}\left(\rho^{\alpha_{0}}(x)\right)\right)}$.

\section{The Optimal-Control Problem}

Our aim in this section is to find the optimal control variable $u(x)$ defined on the $H$-time scale, which minimizes the performance index

$$
J(y, u)=\int_{a}^{b} L\left(x, y^{\rho}(x), u^{\rho}(x), y^{\rho}\left(\rho^{\alpha_{0}}(x)\right), \nabla_{q}^{h} y\left(\rho^{\alpha_{0}}(x)\right)\right) \nabla_{q}^{h} x
$$

subject to the constraint

$$
\nabla_{q}^{h} y(x)=G\left(x, y^{\rho}(x), u^{\rho}(x)\right)
$$

such that

$$
\begin{gathered}
y(b)=c, \quad y(x)=\phi(x) \quad\left(x \in\left[\rho^{\alpha_{0}}(a), a\right]\right), \\
a, b \in \mathbb{T}_{q^{\prime}}^{h} \quad a<\rho^{\alpha_{0}}(b)<b, \\
L:[a, b] \times\left(\mathbb{R}^{n}\right)^{4} \longrightarrow \mathbb{R}, \quad y^{\rho}(x)=y(\rho(x)),
\end{gathered}
$$

where $c$ is a constant and $L$ and $G$ are functions with continuous first and second partial derivatives with respect to all of their arguments. To find the optimal control, we define a modified performance index as

$$
\begin{array}{r}
I(y, u)=\int_{a}^{b}\left[L\left(x, y^{\rho}(x), u^{\rho}(x), y^{\rho}\left(\rho^{\alpha_{0}}(x)\right), \nabla_{q}^{h} y\left(\rho^{\alpha_{0}}(x)\right)\right)\right. \\
\left.+\lambda^{\rho}(x)\left(\nabla_{q}^{h} y(x)-G\left(x, y^{\rho}(x), u^{\rho}(x)\right)\right)\right] \nabla_{q}^{h} x,
\end{array}
$$

where $\lambda$ is a Lagrange multiplier or an adjoint variable. 
Using (3.11) and (3.12) of Theorem 3.2 with $m=3\left(y_{1}=y, y_{2}=u, y_{3}=\lambda\right)$, the necessary conditions for our optimal control are (we remark that as there is no any time-scale derivative of $u(x)$, no boundary constraints for it are needed)

$$
\begin{gathered}
\nabla_{q}^{h} \lambda^{\rho}(x)+q^{-\alpha_{0}} \nabla_{q}^{h} \frac{\partial L}{\partial \nabla_{q}^{h}\left(y\left(\rho^{\alpha_{0}}(x)\right)\right)}\left(\left(\rho^{\alpha_{0}}\right)^{-1}(x)\right)+\lambda^{\rho}(x) \frac{\partial G}{\partial y^{\rho}(x)}-\frac{\partial L}{\partial y^{\rho}(x)} \\
-q^{-\alpha_{0}} \frac{\partial L}{\partial\left(y^{\rho}\left(\rho^{\alpha_{0}}(x)\right)\right)}\left(\left(\rho^{\alpha_{0}}\right)^{-1}(x)\right)=0 \quad\left(x \in\left[a, \rho^{\alpha_{0}}(b)\right]_{\kappa}\right), \\
\nabla_{q}^{h} \lambda^{\rho}(x)+\lambda^{\rho}(x) \frac{\partial G}{\partial y^{\rho}(x)}-\frac{\partial L}{\partial y^{\rho}(x)}=0 \quad\left(x \in\left[\rho^{\alpha_{0}}(b), b\right]_{\kappa}\right), \\
\mathcal{\lambda}^{\rho}(x) \frac{\partial G}{\partial u^{\rho}(x)}-\frac{\partial L}{\partial u^{\rho}(x)}=0 \quad(x \in[a, b]), \\
\left.\frac{\partial L}{\partial \nabla_{q}^{h}\left(y\left(\rho^{\alpha_{0}}(x)\right)\right)}\left(\rho^{\alpha_{0}}\right)^{-1}(x) \eta(x)\right|_{a} ^{\rho^{\alpha_{0}}(b)}=0
\end{gathered}
$$

and also

$$
\nabla_{q}^{h} y(x)=G\left(x, y^{\rho}(x), u^{\rho}(x)\right)
$$

Note that condition (4.6) disappears when the Lagrangian $L$ is free of the delayed time scale derivative of $y$.

\section{The Discrete and Quantum Cases}

We recall that the results in the previous sections are valid for time scales whose backward jump operator $\rho$ has the form $\rho(x)=q x-h$, in particular for the time scale $\mathbb{T}_{q}^{h}$.

\section{(i) The Discrete Case}

If $q=1$ and $h>0$ (of special interest the case when $h=1$ ), then our work becomes on the discrete time scale $h \mathbb{Z}=\{h n: n \in \mathbb{Z}\}$. In this case the functional under optimization will have the form

$$
\begin{gathered}
J^{h}(y)=h \sum_{i=a+1}^{b} L\left(i h, y((i-1) h), \nabla^{h} y(i h), y(i h-(d+1) h), \nabla^{h} y(i h-d h)\right), \\
a, b \in \mathbb{Z}, \quad d \in \mathbb{N}, \quad a<b-d<b,
\end{gathered}
$$

and that $y(b h)=c, y(i h)=\varphi(i h)$ for $a-d \leq i \leq a$ where

$$
\nabla^{h} y(x)=y(x)-y(x-h), \quad x \in h \mathbb{Z}
$$


The necessary condition for $J^{h}(y)$ to possess an extremum for a given function $y:\{i h$ : $i=a-d, a-d+1, \ldots, a, a+1, \ldots, b\} \rightarrow \mathbb{R}^{n}$ is that $y(x)$ satisfies the following $h$-Euler-Lagrange equations:

$$
\begin{gathered}
\nabla^{h} \partial_{2} L(i h)+\nabla^{h} \partial_{4} L((i+d) h)=\partial_{1} L(i h)+\partial_{3} L((i+d) h) \quad(i=a+1, a+2, \ldots, b-d), \\
\nabla^{h} \partial_{2} L(i h)=\partial_{1} L(i h) \quad(i=b-d+1, b-d+2, \ldots, b) .
\end{gathered}
$$

Furthermore, the equation

$$
\partial_{4} L(b h) \eta((b-d) h)-\partial_{4} L((a+d) h) \eta(a h)=0
$$

holds along $y(x)$ for all admissible variations $\eta(x)$ satisfying $\eta(i h)=0, i \in\{a-d, a-d+$ $1, \ldots, a\} \cup\{b\}$.

In this case the $h$-optimal-control problem would read as follows.

Find the optimal control variable $u(x)$ defined on the time scale $h \mathbb{Z}$, which minimizes the $h$-performance index

$$
\begin{gathered}
J^{h}(y, u)=h \sum_{i=a+1}^{b} L\left(i h, y((i-1) h), u((i-1) h), y(i h-(d+1) h), \nabla^{h} y(i h-d h)\right), \\
a, b \in \mathbb{Z}, \quad d \in \mathbb{N}, \quad a<b-d<b
\end{gathered}
$$

subject to the constraint

$$
\nabla^{h} y(i h)=G(i h, y((i-1) h), u((i-1) h)), \quad i=a+1, a+2, \ldots, b
$$

such that

$$
\begin{gathered}
y(b h)=c, \quad y(i h)=\phi(i h) \quad(i=a-d, a-d+1, \ldots, a), \\
a, b \in \mathbb{N}, \quad a<b-d<b .
\end{gathered}
$$


The necessary conditions for this $h$-optimal control are

$$
\begin{gathered}
\nabla^{h} \lambda((i-1) h)+\nabla^{h} \frac{\partial L}{\partial \nabla^{h} y((i-d-1) h)}((i+d) h)+\lambda((i-1) h) \frac{\partial G}{\partial y((i-1) h)} \\
-\frac{\partial L}{\partial y((i-1) h)}-\frac{\partial L}{\partial y((i-d-1) h)}((i+d) h)=0 \quad(i=a+1, a+2, \ldots, b-d), \\
\nabla_{q}^{h} \lambda((i-1) h)+\lambda((i-1) h) \frac{\partial G}{\partial y((i-1) h)}-\frac{\partial L}{\partial y((i-1) h)}=0 \quad(i=b-d+1, b-d+2, \ldots, b), \\
\lambda((i-1) h) \frac{\partial G}{\partial u((i-1) h)}-\frac{\partial L}{\partial u((i-1) h)}=0 \quad(i=a, a+1, \ldots, b), \\
\frac{\partial L}{\partial \nabla^{h} y((i+d) h)}(b h) \eta((b-d) h)-\frac{\partial L}{\partial \nabla^{h} y((i+d) h)}((a+d) h) \eta(a h)=0,
\end{gathered}
$$

and also

$$
\nabla^{h} y(i h)=G(i h, y((i-1) h), u((i-1) h)), \quad i=a+1, a+2, \ldots, b
$$

Note that condition (5.9) disappears when the Lagrangian $L$ is independent of the delayed $\nabla^{h}$ derivative of $y$.

Example 5.1. In order to illustrate our results we analyze an example of physical interest. Namely, let us consider the following discrete action:

$$
J^{h}(t)=\frac{h}{2} \sum_{i=a+1}^{b}\left[\nabla^{h} y(i h)\right]^{2}-V(y(i h-(d+1) h)), \quad a, b \in \mathbb{N}, a<b-d<b
$$

subject to the condition

$$
y(b h)=c, \quad y(i h)=\varphi(i h), \quad \text { for } i=a-d, a-d+1, \ldots, a .
$$

The corresponding $h$-Euler-Lagrange equations are as follows:

$$
\begin{gathered}
y(i h)-2 y((i-1) h)+y((i-2) h)+\frac{\partial V}{\partial y(i h-(d+1) h)}((i+d) h) \quad(i=a+1, \ldots, b-d), \\
y(i h)-2 y((i-1) h)+y((i-2) h)=0 \quad(i=b-d+1, b-d+2, \ldots, b) .
\end{gathered}
$$

We observe that when the delay is removed, that is, $d=0$, the classical discrete EulerLagrange equations are reobtained. 


\section{(ii) The Quantum Case}

If $0<q<1$ and $h=0$, then our work becomes on the time scale $\mathbb{T}_{q}=\left\{q^{n}: n \in \mathbb{Z}\right\} \cup\{0\}$. In this case the functional under optimization will have the form

$$
J_{q}(y)=\int_{a}^{b} L\left(x, y(q x), \nabla_{q} y(x), y\left(x q^{\alpha_{0}+1}\right), \nabla_{q} y\left(x q^{\alpha_{0}}\right)\right) \nabla_{q} x
$$

where

$$
\begin{gathered}
a=q^{\alpha+1}, \quad b=q^{\beta}, \quad \alpha, \beta, \alpha_{0} \in \mathbb{Z}, \alpha>\beta, \beta+\alpha_{0}<\alpha, \\
L:[a, b]_{q} \times\left(\mathbb{R}^{n}\right)^{4} \longrightarrow \mathbb{R}, \quad[a, b]_{q}=\left\{q^{i}: i=\alpha+1, \alpha+2, \ldots, \beta\right\} .
\end{gathered}
$$

Using the $\nabla$-integral theory on time scales, the functional $J_{q}$ in (5.14) turns to be

$$
J_{q}(y)=(1-q) \sum_{i=\alpha}^{\beta} q^{i} L\left(q^{i}, y\left(q^{i+1}\right), \nabla_{q} y\left(q^{i}\right), y\left(q^{\alpha_{0}+i+1}\right), \nabla_{q} y\left(q^{\alpha_{0}+i}\right)\right)
$$

The necessary condition for $J_{q}(y)$ to possess an extremum for a given function $y$ : $\left\{q^{i}: i=\alpha+1-\alpha_{0}, \alpha+2-\alpha_{0} \ldots, \alpha, \alpha+1, \ldots, \beta\right\} \rightarrow \mathbb{R}^{n}$ is that $y(x)$ satisfies the following $q$-Euler-Lagrange equations:

$$
\begin{gathered}
\nabla_{q} \frac{\partial L}{\partial\left(\nabla_{q} y(x)\right)}(x)+q^{-\alpha_{0}} \nabla_{q} \frac{\partial L}{\partial\left(\nabla_{q} y\left(q^{\alpha_{0}} x\right)\right)}\left(q^{-\alpha_{0}} x\right) \\
\quad=\frac{\partial L}{\partial y(q x)}(x)+q^{-\alpha_{0}} \frac{\partial L}{\partial y\left(q^{\alpha_{0}+1} x\right)}\left(q^{-\alpha_{0}} x\right) \quad\left(x \in\left[a, q^{\alpha_{0}} b\right]_{\kappa}\right), \\
\nabla_{q} \frac{\partial L}{\partial\left(\nabla_{q} y(x)\right)}(x)=\frac{\partial L}{\partial(y(q x))}(x) \quad\left(x \in\left[q^{\alpha_{0}} b, b\right]_{\kappa}\right) .
\end{gathered}
$$

Furthermore, the equation

$$
\left.q^{-\alpha_{0}} \frac{\partial L}{\partial\left(\nabla_{q} y\left(\rho^{\alpha_{0}}(x)\right)\right)}\left(q^{-\alpha_{0}} x\right) \eta(x)\right|_{a} ^{q^{\alpha_{0}} b}=0
$$

holds along $y(x)$ for all admissible variations $\eta(x)$ satisfying $\eta(x)=0, x \in\left[q^{\alpha_{0}} a, a\right]_{q} \cup\{b\}$.

In this case the $q$-optimal-control problem would read as follows.

Find the optimal control variable $u(x)$ defined on the $\mathbb{T}_{q}$-time scale, which minimizes the performance index

$$
J_{q}(y, u)=\int_{a}^{b} L\left(x, y(q x), u(q x), y\left(q^{\alpha_{0}+1} x\right), \nabla_{q} y\left(q^{\alpha_{0}+1} x\right)\right) \nabla_{q} x
$$


subject to the constraint

$$
\nabla_{q}^{h} y(x)=G(x, y(q x), u(q x))
$$

such that

$$
\begin{gathered}
y(b)=c, \quad y(x)=\phi(x) \quad\left(x \in\left[q^{\alpha_{0}} a, a\right]\right), \\
a=q^{\alpha+1}, \quad b=q^{\beta}, \quad \alpha_{0}+\beta<\alpha+1, \\
L:[a, b]_{q} \times\left(\mathbb{R}^{n}\right)^{4} \longrightarrow \mathbb{R},
\end{gathered}
$$

where $c$ is a constant and $L$ and $G$ are functions with continuous first and second partial derivatives with respect to all of their arguments.

The necessary conditions for this $q$-optimal control are

$$
\begin{gathered}
\nabla_{q} \lambda(q x)+q^{-\alpha_{0}} \nabla_{q} \frac{\partial L}{\partial \nabla_{q} y\left(q^{\alpha_{0}+1} x\right)}\left(q^{-\alpha_{0}} x\right)+\lambda(q x) \frac{\partial G}{\partial y(q x)}-\frac{\partial L}{\partial y(q x)} \\
-q^{-\alpha_{0}} \frac{\partial L}{\partial y\left(q^{\alpha_{0}+1} x\right)}\left(q^{-\alpha_{0}} x\right)=0 \quad\left(x \in\left[a, q^{\alpha_{0}} b\right]_{\kappa}\right), \\
\nabla_{q} \lambda(q x)+\lambda(q x) \frac{\partial G}{\partial y(q x)}-\frac{\partial L}{\partial y^{\rho}(x)}=0 \quad\left(x \in\left[q^{\alpha_{0}} b, b\right]_{\kappa}\right), \\
\lambda(q x) \frac{\partial G}{\partial u(q x)}-\frac{\partial L}{\partial u(q x)}=0 \quad\left(x \in[a, b]_{q}\right), \\
\left.\frac{\partial L}{\partial \nabla_{q} y\left(q^{\alpha_{0}} x\right)}\left(q^{-\alpha_{0}} x\right) \eta(x)\right|_{a} ^{q^{\alpha_{0}} b}=0,
\end{gathered}
$$

and also

$$
\nabla_{q}^{h} y(x)=G(x, y(q x), u(q x))
$$

Note that condition (5.25) disappears when the Lagrangian $L$ is independent of the delayed $\nabla_{q}$ derivative of $y$.

Example 5.2. Suppose that the problem is that of finding a control function $u(x)$ defined on the time scale $T_{q}$ such that the corresponding solution of the controlled system

$$
\nabla_{q} y(x)=-r y(q x)+u(q x), \quad r>0,
$$

satisfying the conditions

$$
y(b)=c, \quad y(x)=\varphi(x), \quad \text { for } x \in\left[q^{\alpha_{0}} a, a\right]_{q^{\prime}} \quad a=q^{\alpha+1}, \quad b=q^{\beta}, \quad \alpha_{0}+\beta<\alpha+1
$$


is an extremum for the $q$-integral functional ( $q$-quadratic delay cost functional):

$$
J_{q}(y(x), u(x))=\frac{1}{2}(1-q) \sum_{i=\alpha}^{\beta} q^{i}\left[y^{2}\left(q^{i+\alpha_{0}+1}\right)+u^{2}\left(q^{i+1}\right)\right]
$$

According to (5.24) and (5.25), the solution of the problem satisfies

$$
\begin{gathered}
\nabla_{q} \lambda(q x)=r \lambda(q x)+q^{-\alpha_{0}} y(q x) \quad\left(x \in\left[a, q^{\alpha_{0}} b\right]_{\kappa}\right), \\
\nabla_{q} \lambda(q x)=r \lambda(q x) \quad\left(x \in\left[q^{\alpha_{0}} b, b\right]_{\kappa}\right), \\
\lambda(q x)=u(q x) \quad\left(x \in[a, b]_{q}\right),
\end{gathered}
$$

and of course

$$
\nabla_{q} y(x)=-r y(q x)+u(q x)
$$

When the delay is absent (i.e., $\alpha_{0}=0$ ), it can be shown that the above system is reduced to a second-order $q$-difference equation. Namely, reduced to

$$
\nabla_{q}^{2} y(x)+r q\left(\nabla_{q} y\right)(q x)=q\left(r^{2}+1\right) y(q x)+q r \nabla_{q} y(x)
$$

If we solve recursively for this equation in terms of an integer power series by using the initial data, then the resulting solution will tend to the solutions of the second order linear differential equation:

$$
y^{\prime \prime}-\left(r^{2}+1\right) y=0
$$

Clearly the solutions for this equation are $\exp \left(\sqrt{r^{2}+1} x\right)$ and $\exp \left(-\sqrt{r^{2}+1} x\right)$. For details see [16].

\section{Conclusion}

In this paper we have developed an optimal variational problem in the presence of delay on time scales whose backward jumping operators are of the form $\rho(t)=q t-h, q>0, h \geq 0$, called $H$-time scales. Such kinds of time scales unify the discrete, the quantum, and the continuous cases, and hence the obtained results generalized many previously obtained results either in the presence of delay or without. To formulate the necessary conditions for this optimal control problem, we first obtained the Euler-Lagrange equations for one unknown function then generalized to the n-dimensional case. The state variables of the Lagrangian in this case are defined on the $H$-time scale and contain some delays. When $q=1$ and $h=0$ with the existence of delay some of the results in [14] are recovered. When $0<q<1$ and $h=0$ and the delay is absent most of the results in [16] can be reobtained. When $q=1$ and the delay is 
absent some of the results in [15] are reobtained. When the delay is absent and the time scale is free somehow, some of the results in [17] can be recovered as well.

Finally, we would like to mention that we followed the line of nabla time-scale derivatives in this paper, analogous results can be originated if the delta time-scale derivative approach is followed.

\section{Acknowledgment}

This work is partially supported by the Scientific and Technical Research Council of Turkey.

\section{References}

[1] J. F. Rosenblueth, "Systems with time delay in the calculus of variations: a variational approach," IMA Journal of Mathematical Control and Information, vol. 5, no. 2, pp. 125-145, 1988.

[2] L. C. Young, Lectures on the Calculus of Variations and Optimal Control Theory, Saunders, Philadelphia, Pa, USA, 1969.

[3] S. Hilger, "Analysis on measure chains, a unified approach to continuous and discrete calculus," Results in Mathematics, vol. 18, no. 1-2, pp. 18-56, 1990.

[4] M. Bohner and A. Peterson, Dynamic Equations on Time Scales. An Introduction with Application, Birkhäuser, Boston, Mass, USA, 2001.

[5] F. M. Atici, D. C. Biles, and A. Lebedinsky, "An application of time scales to economics," Mathematical and Computer Modelling, vol. 43, no. 7-8, pp. 718-726, 2006.

[6] G. Sh. Guseinov, "Integration on time scales," Journal of Mathematical Analysis and Applications, vol. 285, no. 1, pp. 107-127, 2003.

[7] R. A. C. Ferreira and D. F. M. Torres, "Higher-order calculus of variations on time scales," in Mathematical Control Theory and Finance, pp. 149-159, Springer, Berlin, Germany, 2008.

[8] R. Almeida and D. F. M. Torres, "Isoperimetric problems on time scales with nabla derivatives," Journal of Vibration and Control, vol. 15, no. 6, pp. 951-958, 2009.

[9] M. Bohner, "Calculus of variations on time scales," Dynamic Systems and Applications, vol. 13, no. 3-4, pp. 339-349, 2004.

[10] R. A. C. Ferreira and D. F. M. Torres, "Remarks on the calculus of variations on time scales," International Journal of Ecological Economics E Statistics, vol. 9, no. F07, pp. 65-73, 2007.

[11] A. B. Malinowska and D. F. M. Torres, "Strong minimizers of the calculus of variations on time scales and the Weierstrass condition," to appear in Proceedings of the Estonian Academy of Sciences.

[12] Z. Bartosiewicz and D. F. M. Torres, "Noether's theorem on time scales," Journal of Mathematical Analysis and Applications, vol. 342, no. 2, pp. 1220-1226, 2008.

[13] D. Baleanu, T. Maaraba, and F. Jarad, "Fractional variational principles with delay," Journal of Physics A, vol. 41, no. 31, Article ID 315403, p. 8, 2008.

[14] O. P. Agrawal, J. Gregory, and P. Spector, "A Bliss-type multiplier rule for constrained variational problems with time delay," Journal of Mathematical Analysis and Applications, vol. 210, no. 2, pp. 702711, 1997.

[15] J. A. Cadzow, "Discrete calculus of variations," International Journal of Control, vol. 11, no. 3, pp. 393407, 1970.

[16] G. Bangerezako, "Variational q-calculus," Journal of Mathematical Analysis and Applications, vol. 289, no. 2, pp. 650-665, 2004.

[17] N. Martins and D. F. M. Torres, "Calculus of variations on time scales with nabla derivatives," Nonlinear Analysis: Theory, Method E Applications, vol. 71, no. 12, pp. 763-773, 2008.

[18] F. M. Atici and G. Sh. Guseinov, “On Green's functions and positive solutions for boundary value problems on time scales," Journal of Computational and Applied Mathematics, vol. 141, no. 1-2, pp. 75-99, 2002.

[19] M. Bohner and A. Peterson, Advances in Dynamic Equations on Time Scales, Birkhäuser, Boston, Mass, USA, 2003.

[20] T. Abdeljawad, "A note on the chain rule on time scales," Journal of Arts and Sciences, vol. 9, pp. 1-6, 2008. 